\title{
Patterns of Drug Use and Serum Sodium Concentrations in Older Hospitalized Patients: A Latent Class Analysis Approach
}

\author{
Richard J. Woodman ${ }^{1}$ Karen M. Wood ${ }^{2} \cdot$ Aline Kunnel $^{1} \cdot$ Maneesha Dedigama $^{3}$. \\ Matthew A. Pegoli ${ }^{4}$ Roy L. Soiza ${ }^{5}$ Arduino A. Mangoni ${ }^{3}$
}

Published online: 27 October 2016

(c) The Author(s) 2016. This article is published with open access at Springerlink.com

\begin{abstract}
Background Several drugs may lower serum sodium concentrations $(\mathrm{NaC})$ in older patients. However, distinguishing their individual effects is particularly difficult in this population because of the high prevalence of polypharmacy and disease states that are per se associated with hyponatremia.

Objectives Our objective was to identify specific patterns of medication use in older hospitalized patients and determine whether these patterns were associated with serum $\mathrm{NaC}$.

Methods We collected clinical and demographic data, preadmission drugs, Drug Burden Index (DBI) score, and average $\mathrm{NaC}$ during hospitalization in a consecutive series of older medical patients $(n=101$, mean \pm standard deviation [SD] age $87 \pm 6$ years). We used latent class analysis (LCA) to identify specific patterns of drug use and multivariate regression to determine the associations
\end{abstract}

Arduino A. Mangoni

arduino.mangoni@flinders.edu.au

1 Centre for Epidemiology and Biostatistics, School of Medicine, Flinders University, GPO Box 2100, Adelaide, SA 5001, Australia

2 School of Medicine and Dentistry, University of Aberdeen, Polwarth Building, Foresterhill, Aberdeen AB25 2ZD, UK

3 Department of Clinical Pharmacology, School of Medicine, Flinders University and Flinders Medical Centre, Flinders Drive, Bedford Park, SA 5042, Australia

4 Department of Pharmacy, Flinders Medical Centre, Flinders Drive, Bedford Park, SA 5042, Australia

5 Department of Medicine for the Elderly, NHS Grampian, Aberdeen Royal Infirmary, Foresterhill, Aberdeen AB25 2ZN, UK between 14 separate drug classes, identified patterns of drug use, and $\mathrm{NaC}$.

Results LCA revealed three patterns: lower overall drug use (class 1), anticoagulant use and higher drug use (class 2 ), and antiplatelet use (class 3$)$. Mean $( \pm$ SD) DBI score in each class was $2.7 \pm 1.3,3.3 \pm 1.6$, and $2.4 \pm 1.5$, respectively $(p=0.04)$. Mean $( \pm \mathrm{SD}) \mathrm{NaC}$ in classes 1,2 , and 3 were $140.6 \pm 6.8, \quad 138.7 \pm 5.3, \quad$ and $136.5 \pm 4.7 \mathrm{mmol} / \mathrm{l}, \quad$ respectively $(p=0.006)$. After adjustment for age, sex, Charlson Comorbidity Index score, estimated glomerular filtration rate (eGFR), DBI score, and digoxin use, mean $\mathrm{NaC}$ in class 2 and class 3 was significantly lower than in class $1(-3.9 \mathrm{mmol} / \mathrm{l} ; 95 \%$ confidence interval $[\mathrm{CI}]-7.1$ to $-0.8, p=0.01$ and $-5.2 \mathrm{mmol} / \mathrm{l}$; $95 \%$ CI -7.9 to $-2.5, p<0.001$, respectively). Mean serum $\mathrm{NaC}$ was not significantly associated with any of the 14 individually assessed drug classes. In addition to latent class, increasing age and higher eGFR were also independently associated with lower serum $\mathrm{NaC}(p=0.002$ and $p=0.03$, respectively).

Conclusion LCA enabled us to identify patterns of drug use associated with lower serum $\mathrm{NaC}$ in older inpatients. Our results suggest that older patients using antiplatelets or anticoagulants are especially at risk of lower serum $\mathrm{NaC}$. 


\section{Key Points}

Distinguishing the individual effects of drugs on serum sodium $(\mathrm{Na})$ concentrations in older inpatients is difficult because of the high prevalence of polypharmacy and disease states that are per se associated with hyponatremia.

To investigate associations between drug use, clinical characteristics, and serum $\mathrm{Na}$ concentrations in older inpatients, we applied a clustering technique known as latent class analysis (LCA) to identify specific patterns of drug use.

By identifying patterns of drug use with LCA, we established that age, eGFR, antiplatelet drugs, and anticoagulants are independently associated with lower serum $\mathrm{Na}$ concentrations.

\section{Introduction}

Serum sodium concentrations $(\mathrm{NaC})<135 \mathrm{mmol} / \mathrm{l}$ (hyponatremia) are observed in up to $50 \%$ of older patients admitted to hospital [1,2]. The high prevalence of this electrolyte abnormality in older hospitalized patients might be secondary to the presence of one, or more, disease states characterized by alterations in sodium and water balance, impaired homeostasis, and polypharmacy, as well as inhospital iatrogenic interventions [3]. There is increasing evidence that lower $\mathrm{NaC}$ values are independently associated with adverse clinical outcomes in old age [3]. More specifically, they predict loss of independence, increased length of stay, and mortality in older hospitalized patients [4-6].

Several drugs and drug classes, e.g., diuretics, antidepressants, and antiepileptics, are known to reduce $\mathrm{NaC}$. Proposed mechanisms for these reductions include alterations in sodium and water homeostasis, potentiation of the physiological effects of the antidiuretic hormone, and resetting of control mechanisms located in the hypothalamus [7]. However, it is possible that associations between $\mathrm{NaC}$ and the use of diuretics, antidepressants, antiepileptics, and other drug classes are at least partly explained by the disease state for which these drugs are prescribed in the first instance [8].

The increased risk of hyponatremia, an independent predictor of adverse outcomes in the older population, with several commonly prescribed drugs warrants regular medication reviews to prevent and rectify this electrolyte abnormality, potentially improving outcomes in this group [3]. However, investigating associations between drugs and $\mathrm{NaC}$ in patients with concomitant prescribed medications and multiple disease states presents significant challenges. In particular, it is extremely difficult to disentangle the independent effects on $\mathrm{NaC}$ of individual drugs and other clinical and demographic parameters [7]. One methodological approach that might be useful in simplifying the problem of trying to identify likely associations between drugs and $\mathrm{NaC}$ is to group individuals by their most distinctive patterns of medication use, typically using two to four groups to make interpretation easier. If examination of the resulting classes reveals a very high probability of either using or avoiding a particular medication, this raises the possibility that any observed differences in clinical characteristics (such as $\mathrm{NaC}$ ) between the classes may partly be due to use of that medication. Such an approach also helps increase the external validity and clinical relevance of the study since, in the real world, older patients generally take a multitude of medications, which is captured in the resulting groups.

To investigate the independent associations between drug use and $\mathrm{NaC}$ in older hospitalized patients, we applied a clustering technique known as latent class analysis (LCA) to identify specific patterns of drug use. We then determined whether these patterns were associated with lower $\mathrm{NaC}$.

\section{Methods}

\subsection{Study Population}

We retrospectively studied a consecutive series of 101 patients aged $\geq 75$ years who were admitted to general medicine wards at Flinders Medical Centre, a 450-bed metropolitan teaching hospital in the Southern Adelaide Local Health Network, Adelaide, Australia, between 30 October 2014 and 1 May 2015 and subsequently discharged to a residential aged care facility. The study was approved by the Southern Adelaide Clinical Human Research Ethics Committee (Ethics Approval Number: 457.14).

\subsection{Clinical and Demographic Variables}

Data on age, sex, and comorbidities were collected from individual patient's clinical notes, discharge summaries, and pathology results. This information was used to calculate the Charlson Comorbidity Index (CCI) score for each patient. The CCI, widely used in clinical research as a measure of comorbidity and a prognostic indicator for survival, encompasses 19 medical 
conditions weighted with a score of 1 to 6 , with total scores ranging from 0 to 37 [9].

\subsection{Medication Exposure}

Medication exposure at the time of hospital presentation was characterized as type of medication, total number of medications, and Drug Burden Index (DBI) score. A comprehensive list of all medications on admission was formulated by a pharmacist. This involved a minimum of three sources of information (e.g., patient interview, carer/ relative interview, phone call to community pharmacy, general practitioner medication list, patient's own medications, phone call to aged care facility manager). The DBI score was also assessed as an established measure of exposure to drugs with sedative and/or anticholinergic effects, which includes several drugs associated with an increased risk of hyponatremia [7, 10, 11]. The drug burden (DB) was calculated for each patient according to the following equation:

$\mathrm{DB}=B_{\mathrm{AC}}+B_{\mathrm{S}}$

where $B_{\mathrm{AC}}$ and $B_{\mathrm{S}}$ indicate the burden from anticholinergic and sedative drugs, respectively. The DBI for each anticholinergic or sedative drug was calculated using the following equation, as previously described [10]:

$\mathrm{DBI}=\sum[D /(\delta+D)]$

where $D$ is the daily dose and $\delta$ is the minimum recommended daily dose approved by the Australasian Society of Clinical and Experimental Pharmacologists and Physiologists, the Royal Australasian College of General Practitioners, and the Pharmaceutical Society of Australia (Australian Medicines Handbook). The minimum recommended daily dose was used as an estimate of the dose required to achieve $50 \%$ of the maximum anticholinergic or sedative effect. Complementary medications, topical medications, or medications instructed to be taken as required were excluded from the DBI calculations.

\subsection{Biochemical Parameters}

Data on $\mathrm{NaC}$ and estimated glomerular filtration rate (eGFR) were captured from the hospital electronic database (OACIS Clinical Care Suite Safety Learning System, Government of South Australia). Patients were regularly assessed for $\mathrm{NaC}$ and eGFR throughout their hospital stay as part of their routine clinical assessment. The number of individual tests varied according to the reason for admission and length of hospital stay. For analysis purposes, we used the mean $\mathrm{NaC}$ and eGFR across all of their assessments.

\subsection{Statistical Analysis}

We performed an LCA based on the use or not of 14 separate commonly used drug classes (listed in Table 1). The LCA thus grouped patients according to their most specific patterns of drug use, and their class membership was decided by their highest (posterior) predicted probability of class membership. We used the Akaike Information Criterion (AIC) statistic to assess the model fit for three different models, specifying two, three, or four latent classes. Following the LCA, the distinctive characteristics of each class was determined based on the observed drug use distributions, and an appropriate label was then assigned to each class. We then performed several different analyses based on the newly formed latent classes. First, we compared differences across the latent class groups in patient characteristics and individual drug use in univariate analysis, using one-way analysis of variation (ANOVA) for continuous variables and Fisher's exact test for categorical variables. Then, to establish whether differences in $\mathrm{NaC}$ based on medication use could also be revealed using a more standard regression approach, we used multivariate linear regression to compare mean $\mathrm{NaC}$ for users/non-users of each medication class after adjustment for age, sex, CCI score, DBI score, and eGFR, which were each considered to be potential confounders in the relationship between $\mathrm{NaC}$ and medication use. The estimated marginal mean differences between users/non-users were calculated and assessed for significance. We also performed an additional multivariate analysis for mean $\mathrm{NaC}$ and each medication excluding the DBI score from the adjustment. Results were substantively the same and are not reported. A similar analysis was performed to compare differences in mean $\mathrm{NaC}$ between latent classes, with models that were unadjusted (model 1), and adjusted for age, sex, CCI and DBI scores, and eGFR (model 2). In a final model (model 3), we also adjusted for digoxin use, since patients with heart failure are known to have lower $\mathrm{NaC}$. Lower eGFR and digoxin use are indicators of renal and heart failure, respectively, which may also affect $\mathrm{NaC}$ and confound potential medication use/ $\mathrm{NaC}$ associations. Finally, we performed multinomial logistic regression with latent class membership as the nominal categorical dependent variable and $\mathrm{NaC}$ for each individual as the exposure variable of interest. This is a standard approach following LCA; it enables calculation of the estimated probabilities of class membership for any given $\mathrm{NaC}$ and plotting of the marginal posterior probabilities against each other. The analysis of $\mathrm{NaC}$ was based on mean values for each individual across their hospital stay, which ranged from one to ten tests. Similarly, the analysis of eGFR was also based on the mean eGFR values during 
Table 1 Clinical characteristics according to latent medication use class membership

\begin{tabular}{|c|c|c|c|c|}
\hline Characteristic & $\begin{array}{l}\text { Class 1: lower medication use } \\
(N=27)\end{array}$ & $\begin{array}{l}\text { Class 2: anticoagulant users } \\
(N=28)\end{array}$ & $\begin{array}{l}\text { Class 3: antiplatelet users } \\
(N=46)\end{array}$ & $p$ value $^{\mathrm{a}}$ \\
\hline Males & $11(40.7)$ & $10(35.7)$ & $19(41.3)$ & 0.90 \\
\hline \multicolumn{4}{|l|}{ Admitted from } & \multirow[t]{3}{*}{0.81} \\
\hline Home & $12(44.4)$ & $14(50.0)$ & $24(52.2)$ & \\
\hline Aged care facility & $15(55.6)$ & $14(50.0)$ & $22(47.8)$ & \\
\hline Age, years & $89 \pm 7$ & $86 \pm 6$ & $87 \pm 5$ & 0.18 \\
\hline CCI score & $2.9 \pm 2.1$ & $3.9 \pm 2.1$ & $2.8 \pm 1.7$ & 0.04 \\
\hline eGFR (ml/min) & $49 \pm 23$ & $44 \pm 24$ & $53 \pm 21$ & 0.85 \\
\hline $\mathrm{NaC}(\mathrm{mmol} / \mathrm{l})$ & $140.6 \pm 6.8$ & $138.7 \pm 5.3$ & $136.5 \pm 4.7$ & 0.006 \\
\hline $\mathrm{NaC}<135 \mathrm{mmol} / \mathrm{l}^{\mathrm{b}}$ & $7(25.9)$ & $8(28.6)$ & $23(50.0)$ & 0.07 \\
\hline $\mathrm{NaC}<135 \mathrm{mmol} / \mathrm{l}^{\mathrm{c}}$ & $8(30)$ & $9(32)$ & $23(50)$ & 0.16 \\
\hline DBI & $2.70 \pm 1.35$ & $3.32 \pm 1.59$ & $2.41 \pm 1.48$ & 0.04 \\
\hline Medications used & $2(0-5)$ & $5(4-9)$ & $4(2-8)$ & $<0.001$ \\
\hline Number of $\mathrm{NaC}$ tests & $4.6 \pm 3.0$ & $5.0 \pm 2.9$ & $5.2 \pm 3.0$ & 0.70 \\
\hline Number of eGFR tests & $3.7 \pm 3.0$ & $4.6 \pm 3.2$ & $5.0 \pm 3.0$ & 0.25 \\
\hline \multicolumn{5}{|c|}{ Individual medication use } \\
\hline Antiplatelets & $3(11.1)$ & $2(7.1)$ & $45(97.8)$ & $<0.001$ \\
\hline Anticoagulants & $0(0.0)$ & $28(100.0)$ & $1(2.2)$ & $<0.001$ \\
\hline Statins & $2(7.4)$ & $16(57.1)$ & $18(39.1)$ & $<0.001$ \\
\hline $\begin{array}{l}\text { Proton pump } \\
\text { inhibitors }\end{array}$ & $11(40.7)$ & $18(64.3)$ & $22(47.8)$ & 0.20 \\
\hline Digoxin & $1(3.7)$ & $6(21.4)$ & $8(17.4)$ & 0.13 \\
\hline Oral hypoglycemics & $0(0.0)$ & $7(25.0)$ & $6(13.0)$ & 0.01 \\
\hline Bisphosphonates & $0(0.0)$ & $3(10.7)$ & $7(15.2)$ & 0.11 \\
\hline Benzodiazepines & $2(7.4)$ & $7(25.0)$ & $4(8.7)$ & 0.10 \\
\hline Diuretics & $7(25.9)$ & $20(71.4)$ & $21(45.6)$ & 0.003 \\
\hline ACEI/ARB & $7(25.9)$ & $7(25.0)$ & $16(34.8)$ & 0.64 \\
\hline Beta-blockers & $6(22.2)$ & $21(75.0)$ & $17(37.0)$ & $<0.001$ \\
\hline Antipsychotics & $0(0.0)$ & $0(0.0)$ & $6(13.1)$ & 0.03 \\
\hline Opioids & $6(22.2)$ & $6(21.4)$ & $7(15.2)$ & 0.68 \\
\hline Antidepressants & $15(55.6)$ & $20(71.4$ & $23(50.0)$ & 0.19 \\
\hline
\end{tabular}

Data are presented as mean \pm standard deviation, $n(\%)$, or median (range)

$A C E I$ angiotensin-converting enzyme inhibitor, ANOVA analysis of variance, $A R B$ angiotensin receptor blocker, CCI Charlson Comorbidity Index, $D B I$ Drug Burden Index, eGFR estimated glomerular filtration rate, $\mathrm{NaC}$ serum sodium concentration

${ }^{a}$ Using Fishers exact test for categorical variables and one-way ANOVA for continuous variables

b $\mathrm{NaC}$ on admission to hospital

c Period prevalence from $\mathrm{NaC}$ values during hospital stay

hospital stay, which ranged from one to ten except for eight individuals who were not assessed. The LCA was performed using the poLCA package in R software, which performs LCA for polytomous outcome variables. All other analysis was performed using STATA (StataCorp, version 14.1, College Station, TX, USA). A type 1 error rate of $\alpha=0.05$ was considered statistically significant for all analyses.

\section{Results}

Medication use for each of the 14 listed drug classes (Tables 1,2) was completed for all subjects, and the LCA was therefore based on all 101 subjects. Mean eGFR data were missing for seven subjects who were therefore not included in the multivariate regression models, which were analysed using 94 subjects (Table 3). 
Table 2 Observed mean sodium concentrations by nonuse/use of individual medications $(n=101)$

\begin{tabular}{llll}
\hline Medication (no. of users) & Non-users & Users & Users vs. non-users $(p \text { value })^{\mathrm{a}}$ \\
\hline Antiplatelets $(n=50)$ & $139.3 \pm 6.0$ & $137.2 \pm 5.2$ & $-1.9 \pm 1.2(0.11)$ \\
Anticoagulants $(n=29)$ & $138.1 \pm 5.9$ & $138.6 \pm 5.2$ & $-0.9 \pm 1.4(0.50)$ \\
Statins $(n=36)$ & $137.8 \pm 6.2$ & $139.0 \pm 4.6$ & $-0.6 \pm 1.3(0.68)$ \\
PPI $(n=51)$ & $138.9 \pm 5.9$ & $137.6 \pm 5.5$ & $-1.9 \pm 1.2(0.12)$ \\
Digoxin $(n=15)$ & $138.1 \pm 5.9$ & $139.0 \pm 4.2$ & $1.3 \pm 1.7(0.44)$ \\
Oral hypoglycemics $(n=13)$ & $138.3 \pm 5.8$ & $138.0 \pm 4.7$ & $-2.5 \pm 1.8(0.16)$ \\
Bisphosphonates $(n=10)$ & $138.2 \pm 5.8$ & $138.8 \pm 4.7$ & $-0.6 \pm 2.1(0.75)$ \\
Benzodiazepines $(n=13)$ & $138.3 \pm 5.9$ & $138.1 \pm 3.5$ & $-0.5 \pm 2.0(0.79)$ \\
Diuretics $(n=48)$ & $138.5 \pm 6.2$ & $138.0 \pm 5.0$ & $-1.1 \pm 1.2(0.38)$ \\
ACEI/ARB $(n=30)$ & $138.7 \pm 6.1$ & $137.2 \pm 4.4$ & $-1.3 \pm 1.3(0.31)$ \\
Beta-blockers $(n=44)$ & $138.4 \pm 5.9$ & $138.0 \pm 5.4$ & $-1.5 \pm 1.2(0.23)$ \\
Antipsychotics $(n=6)$ & $138.5 \pm 5.7$ & $134.7 \pm 4.6$ & $-3.3 \pm 2.4(0.16)$ \\
Opioids $(n=19)$ & $137.8 \pm 5.6$ & $140.3 \pm 5.8$ & $2.5 \pm 1.7(0.15)$ \\
Antidepressants $(n=58)$ & $138.7 \pm 4.9$ & $137.9 \pm 6.2$ & $-0.9 \pm 1.2(0.46)$ \\
\hline
\end{tabular}

Data are presented as observed mean \pm standard deviation

$A C E I$ angiotensin-converting enzyme inhibitor, $A R B$ angiotensin receptor blocker, PPI proton pump inhibitor

${ }^{a}$ Marginal mean difference and associated $p$ value for users vs. non-users using multivariate linear regression adjusted for age, sex, Charlson Comorbidity Index score, Drug Burden Index, and estimated glomerular filtration rate

Table 3 Results from simple $(n=101)$ and multivariate $(n=94)$ linear regression showing estimated differences in mean serum sodium concentrations $(\mathrm{mmol} / \mathrm{l})$ by latent pattern of medication use

\begin{tabular}{|c|c|c|c|c|c|c|}
\hline & Model 1 & $p$ value & Model 2 & $p$ value & Model 3 & $p$ value \\
\hline \multicolumn{7}{|l|}{ Latent class } \\
\hline Class 1 (lower medications) & Reference & - & Reference & - & Reference & - \\
\hline Class 2 (anticoagulants) & $-2.0(-4.9$ to 1.0$)$ & 0.18 & $-3.5(-6.6$ to -0.4$)$ & $\mathbf{0 . 0 3}$ & $-3.9(-7.1$ to -0.8$)$ & 0.01 \\
\hline Class 3 (antiplatelets) & $-4.3(-6.9$ to -1.6$)$ & 0.002 & $-4.7(-7.4$ to -2.1$)$ & 0.001 & $-5.2(-7.9$ to -2.5$)$ & $<0.001$ \\
\hline Age, years & & & $-0.34(-0.56$ to -0.13$)$ & 0.002 & $-0.34(-0.55$ to -0.13$)$ & 0.002 \\
\hline Charlson comorbidity score & & & $-0.16(-0.75$ to 0.43$)$ & 0.60 & $-0.17(-0.76$ to 0.42$)$ & 0.56 \\
\hline Male vs. female & & & $-0.8(-3.1$ to 1.6$)$ & 0.51 & $-0.6(-3.0$ to 1.7$)$ & 0.58 \\
\hline \multicolumn{7}{|l|}{ eGFR category (ml/min) } \\
\hline $0-29$ & & & Reference & - & Reference & - \\
\hline $30-59$ & & & $-3.1(-6.3$ to 0.0$)$ & 0.05 & $-3.2(-6.3$ to -0.0$)$ & 0.048 \\
\hline $60-90$ & & & $-4.3(-7.6$ to -1.0$)$ & 0.01 & $-4.3(-7.6$ to -1.1$)$ & 0.01 \\
\hline Drug burden index & & & $0.16(-0.61$ to 0.93$)$ & 0.69 & $0.1(-0.6$ to 0.9$)$ & 0.75 \\
\hline Digoxin use (yes vs. no) & & & & & $2.5(-0.7$ to 5.7$)$ & 0.13 \\
\hline
\end{tabular}

Data are presented as $\beta$ (95\% confidence interval) unless otherwise indicated. Model 1: unadjusted analysis. Model 2: model 1 plus adjustment for age, sex, Charlson Comorbidity Index score, Drug Burden Index, and eGFR. Model 3: model 2 plus adjustment for digoxin use

Significant $p$ values are in bold

$e G F R$ estimated glomerular filtration rate

\subsection{Latent Class Analysis}

The AIC statistic for the LCA models with two, three, and four classes was 1499.6, 1500.9, and 1505.4, respectively. Although the optimal number of classes was therefore two based on using the smallest AIC for best model fit, we chose to use three classes since each of the three classes had a distinctive medication class use pattern. These patterns were characterized as follows: lower medication use (class 1), anticoagulant users combined with higher overall medication use (class 2), and antiplatelet users (class 3). 


\subsection{Clinical Characteristics}

Table 1 displays the clinical characteristics of the patients according to their latent class membership. There were no differences between groups in sex, age, and eGFR. The CCI score was highest amongst subjects in class 2. This class also had a significantly higher DBI score than the other two classes $(p=0.04)$. The median number of medications used in classes 1,2 , and 3 was two, five, and four, respectively $(p<0.001)$. The use of statins, betablockers, and diuretics were all significantly higher in class 2 than in the other two classes.

\subsection{Serum Sodium (Na) Concentration: Univariate Analysis}

The mean ( \pm standard deviation $[\mathrm{SD}])$ number of $\mathrm{NaC}$ assessments during hospital stay for the 101 subjects with at least one test was $5 \pm 3$ (range 1-10). In univariate analysis, there was no difference in the number of assessments performed across the three latent classes. However, mean $\mathrm{NaC}$ decreased significantly across classes $(p=0.006)$ (Table 1): it was significantly lower for class 3 than for class $1(p=0.002)$ but not class $2(p=0.18)$.

\subsection{Serum Na Concentration: Multivariate Analysis}

Table 2 shows the observed mean $( \pm \mathrm{SD}) \mathrm{NaC}$ for nonusers and users of each of the 14 medication classes. After adjustment for age, sex, CCI and DBI score, and eGFR, the adjusted marginal mean differences were not significantly different between classes for any of the separate medication classes. Table 3 shows the estimated coefficients from the multivariate linear regression, which included latent class membership rather than individual drug classes. After adjustment for age, sex, CCI and DBI score, and eGFR (model 2), the mean $\mathrm{NaC}$ among patients in class 2 and class 3 were significantly lower than among those in class 1 ( $-3.5 \mathrm{mmol} / \mathrm{l} ; 95 \%$ confidence interval $[\mathrm{CI}]-6.6$ to -0.4 , $p=0.03$ and $-4.7 \mathrm{mmol} / \mathrm{l} ; 95 \%$ CI -7.4 to -2.1 , $p=0.001$, respectively). The additional adjustment for digoxin use in model 3 increased the differences for class 2 and class 3 versus class 1 ( $-3.9 \mathrm{mmol} / \mathrm{l} ; 95 \% \mathrm{CI}-7.0$ to $0.8, p=0.02$ and $-5.2 \mathrm{mmol} / \mathrm{l} ; 95 \% \mathrm{CI}-7.9$ to -2.5 , $p<0.001$, respectively). Increasing age and higher eGFR were also independently associated with lower $\mathrm{NaC}$ ( $p=0.002$ and $p=0.03$, respectively). We also performed sensitivity analyses in which we used $\mathrm{NaC}$ on admission as the outcome rather than the mean values for each individual across their hospital stay. The pattern of results was similar, and the differences for class 2 and class 3 versus class 1 was slightly larger $(-5.5 \mathrm{mmol} / \mathrm{l} ; 95 \%$ CI -9.7 to $-1.2, p=0.01$ and $-6.9 \mathrm{mmol} / \mathrm{l} ; 95 \% \mathrm{CI}-10.6$

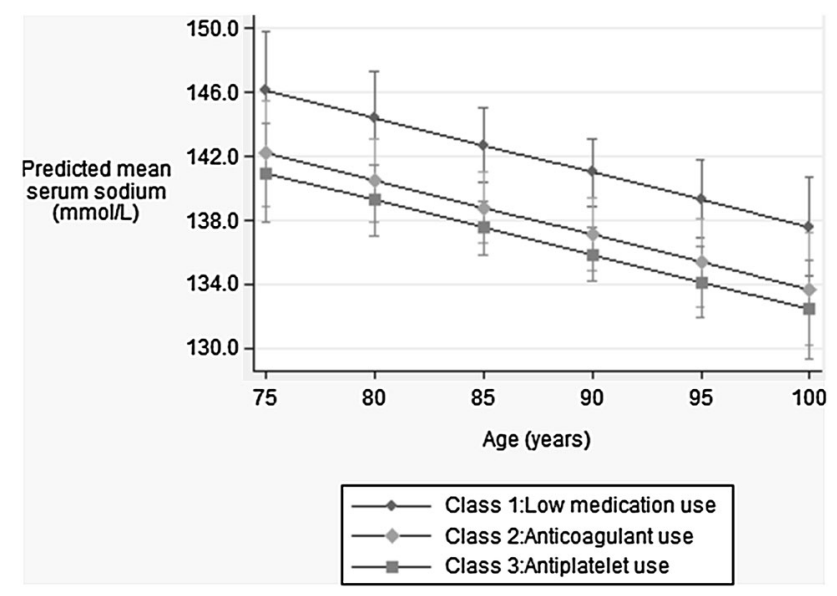

Fig. 1 Marginal mean serum sodium concentration (mmol/l) by age and latent class of medication use. Mean was calculated using multivariate linear regression and adjusted for age, sex, Charlson Comorbidity Index score, Drug Burden Index, estimated glomerular filtration rate, and digoxin use

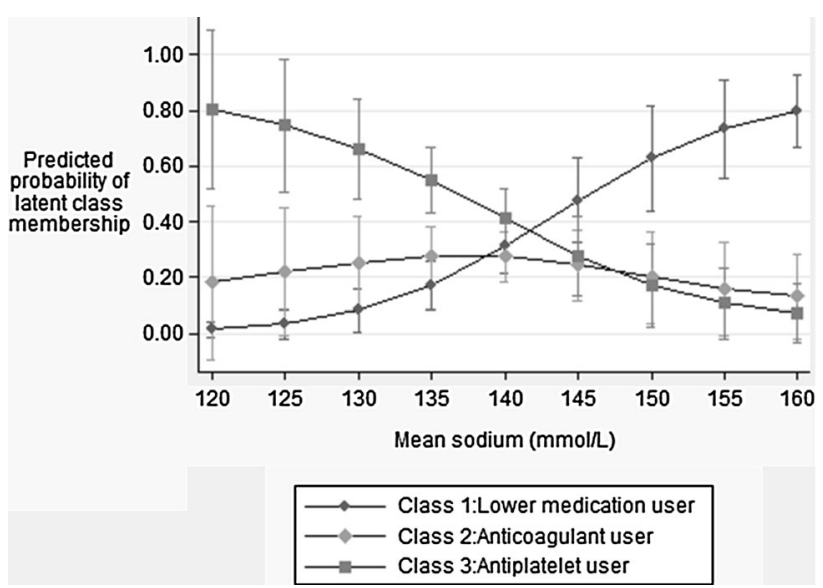

Fig. 2 Probability of latent class membership by mean serum sodium concentration during hospital admission. Probabilities are for membership in each particular class for a given serum sodium concentration; the sum of the three probabilities at each serum sodium concentration sum to 1 . The multinomial logistic regression model was adjusted for age, sex, Charlson Comorbidity Index, estimated glomerular filtration rate, and Drug Burden Index. Vertical bars represent $95 \%$ confidence intervals

to $-3.3, p<0.001$, respectively). Figure 1 shows the adjusted marginal mean $\mathrm{NaC}$ and $95 \%$ CIs according to age and class membership.

\subsection{Class Membership Probabilities}

In multinomial logistic regression after adjustment for the predictors in model 3 above, patients with lower $\mathrm{NaC}$ were significantly less likely to be within class 1 (low medication use) than within class 2 (anticoagulant users, odds ratio [OR] $0.87,95 \%$ CI $0.76-0.99, p=0.03$ ) or class 3 (antiplatelet users, OR $0.82,95 \%$ CI $0.72-0.92, p=0.001$ ). 
Figure 2 shows the predicted probabilities of class membership for each given $\mathrm{NaC}$. This shows, for example, that a patient with an $\mathrm{NaC}$ of approximately $120 \mathrm{mmol} / \mathrm{l}$ and other covariates held constant has an approximately $80 \%$ probability of being an antiplatelet user.

\section{Discussion}

We used LCA to characterize patterns of drug use in a consecutive series of older patients admitted to general medicine wards and subsequently discharged to an aged care facility. We observed significant differences in $\mathrm{NaC}$ according to the pattern of medication use. Patterns that reflected a high probability of antiplatelet or anticoagulant medication use had lower $\mathrm{NaC}$ values than patterns of lower overall medication anticoagulant use. Although patients who used anticoagulants generally had a slightly higher overall pattern of use and reduced likelihood of antiplatelet or medication use, our findings remained consistent after adjusting for DBI and CCI score, age, eGFR, and digoxin use. In addition, age, CCI score, and eGFR were also independently associated with $\mathrm{NaC}$, which strengthens the validity of the study given that these factors are all known confounders. The similar results obtained when assessing $\mathrm{NaC}$ on admission rather than the mean concentrations during hospitalization further supports the presence of independent associations between lower $\mathrm{NaC}$ and antiplatelet drugs and anticoagulants. Our approach enabled us to take account for the general background of multiple medication use and comorbidities that typically exists among older patients. It also revealed significant associations that could not be identified when these medications were assessed for association in isolation.

A number of reviews have elegantly discussed the available evidence of the causative role of several drugs on the risk of hyponatremia, particularly in older patients $[3,7,12]$. However, a substantial number of studies (1) comprised small population groups and case reports, (2) included patients only receiving the investigated drug, and (3) did not ascertain the potential contributing role of other clinical and demographic characteristics on the incidence of hyponatremia [3, 7, 12]. These issues are particularly relevant in older patients with multiple disease states and polypharmacy.

We observed significant independent associations between lower $\mathrm{NaC}$ and the patterns of drug use characterized by the concomitant use of antiplatelet and anticoagulant drugs. By contrast, no associations were observed with drugs previously reported as causing hyponatremia, such as proton pump inhibitors, oral hypoglycemics, diuretics, inhibitors of the renin-angiotensin system, antipsychotics, and antidepressants [13-18]. To the best of our knowledge, this is the first report to describe an independent association between patterns of drug use dominated by the use of antiplatelet drugs and anticoagulants and lower $\mathrm{NaC}$. Both classes of drugs are routinely prescribed in primary and secondary cardiovascular prevention as well as in other pro-thrombotic states. The high prevalence of disease states associated per se with lower $\mathrm{NaC}$ in patients prescribed either antiplatelet drugs or anticoagulants, e.g., heart failure, renal failure, and fluid overload, might potentially explain our findings. In other words, the association between $\mathrm{NaC}$ and antiplatelet drugs and anticoagulants might represent confounding by indication. However, this hypothesis is not supported by the lack of associations observed in our study between lower $\mathrm{NaC}$ and other drugs often co-prescribed with antiplatelet drugs and/or anticoagulants, e.g., statins, oral hypoglycemics, diuretics, inhibitors of the renin-angiotensin system, and beta-blockers. Confirmation is required in larger studies in different populations, and further research is also required to identify the mechanisms responsible for a possible effect of antiplatelet drugs and anticoagulants on sodium homeostasis.

Increasing age and higher eGFR were also associated with lower $\mathrm{NaC}$. A negative association between age and $\mathrm{NaC}$ has been previously reported in older patients presenting to hospital. In multivariate regression analysis, Lindner et al. [19] observed that both age $>60$ years (OR $2.5,95 \%$ CI 1.9-3.0, $p<0.001$ ) and use of diuretics (OR $1.9,95 \%$ CI $1.7-2.2, p<0.001)$ were independently associated with hyponatremia in 20,667 patients attending the emergency department. In this study, the strong negative association between age and $\mathrm{NaC}$ was also observed in patients not treated with diuretics [19]. It is therefore possible that ageing per se causes alterations of sodium and water homeostasis either peripherally, e.g., kidney, or centrally, e.g., osmotic receptors in the hypothalamus [20]. Chronic kidney disease, hence a reduced eGFR, is associated with an increased incidence of either hypo- or hypernatremia [21]. A large population study by Kovesdy et al. [22] investigated the prevalence of hyponatremia and hypernatremia in 655,493 US veterans according to the different stages of chronic kidney disease. Overall, the prevalence of hyponatremia was higher than that of hypernatremia (13.5 vs. $2.0 \%$ ). Interestingly, the relative prevalence of hyponatremia was higher in the early stages of chronic kidney disease, whereas the prevalence of hypernatremia was higher in the more advanced stages [22]. Unlike our study, Kovesdy et al. [22] included both inpatient and outpatient populations. Moreover, no multivariate analysis was performed to assess independent associations between eGFR and $\mathrm{NaC}$. Further studies in larger patient populations are required to confirm the presence of an independent association between higher eGFR and hyponatremia in older hospitalized patients. 
Our conclusions rely partly on being able to accurately characterize the three latent classes that were first determined based on statistical analysis alone. Although there was some overlap in medication use across classes, almost all subjects in class 2 used anticoagulants, almost all subjects in class 3 used antiplatelets, no subjects in class 1 used anticoagulants, and only three in class 1 used antiplatelets. Therefore, identifying the main distinguishing feature of each class was straightforward, increasing the likelihood that the observed differences in $\mathrm{NaC}$ between classes may have been due to differences in the use of these two medications.

The study has a number of limitations. Only a fairly limited number of clinical variables were assessed. Therefore, the possibility of unobserved residual confounding remains. In addition, we did not have information on the primary diagnosis at presentation. Although the latter might have affected $\mathrm{NaC}$ per se during hospitalization, this would only cause biased estimates for the associations if there was an imbalance in conditions associated with lower $\mathrm{NaC}$ across the three classes. In addition, although all subjects in class 2 used anticoagulants, they also had a slightly higher medication use overall. However, adjusting for either the number of medications used or the DBI score did not alter our findings. Our sample size was fairly small, and information on eGFR was missing for some subjects. This means that a larger sample size may have enabled us to observe a significant difference in $\mathrm{NaC}$ between individuals in classes 2 and 3. However, the mean marginal difference of approximately $1.1 \mathrm{mmol} / \mathrm{l}$ is unlikely to be clinically significant. We have no data on how and when medications were changed after admission or how prescribing changes affected $\mathrm{NaC}$ and vice versa. Similarly, we were unable to discriminate between shortterm and chronic use medications upon admission. However, our sensitivity analyses on $\mathrm{NaC}$ on admission confirm the presence of independent associations between lower $\mathrm{NaC}$ and antiplatelet drugs and anticoagulants. A further limitation was that we conducted our study in a single hospital using admissions between November and April, inclusively. Therefore, our results need to be replicated in larger multicentre studies, using admissions from a nonsummer period, before they can be made more generalizable. Finally, since our study was cross-sectional in nature, we cannot be sure of whether or not the observed associations were causal. However, it is unlikely that low $\mathrm{NaC}$ or a disease state associated with hyponatremia would be an indicator for the use of antiplatelet drugs or anticoagulants.

The study also has a number of strengths. Attempting to assess the impact of individual medications on $\mathrm{NaC}$ is often problematic because of the observational nature of studies as well as the presence of polypharmacy and multiple disease states in older patients. Using LCA allows a 'subject-focused' as opposed to a 'variable-based' approach, which tries to assess the impact of individual variables. We were also able to use multiple measures of $\mathrm{NaC}$ for each individual patient, thereby increasing the accuracy of the true underlying $\mathrm{NaC}$ for each individual. Finally, we were able to adjust for several important clinical and demographic variables that may influence the association between medication use and $\mathrm{NaC}$, including age and eGFR, which were also associated with $\mathrm{NaC}$.

\section{Conclusion}

Our results are the first to show a substantially higher likelihood of lower $\mathrm{NaC}$ among patients using either antiplatelet drugs or anticoagulants combined with higher medication use in general. Further studies are needed to replicate our findings and to determine the impact of these medications when taken in isolation and when used by a younger population over time.

\section{Compliance with Ethical Standards}

Funding No funding was received for the conduct of this study.

Conflicts of interest RJW, KMW, AK, MD, MAP, RLS, and AAM have no conflicts of interest.

Open Access This article is distributed under the terms of the Creative Commons Attribution-NonCommercial 4.0 International License (http://creativecommons.org/licenses/by-nc/4.0/), which permits any noncommercial use, distribution, and reproduction in any medium, provided you give appropriate credit to the original author(s) and the source, provide a link to the Creative Commons license, and indicate if changes were made.

\section{References}

1. Mannesse CK, Vondeling AM, van Marum RJ, van Solinge WW, Egberts TC, Jansen PA. Prevalence of hyponatremia on geriatric wards compared to other settings over four decades: a systematic review. Ageing Res Rev. 2013;12(1):165-73. doi:10.1016/j.arr. 2012.04.006.

2. Hoyle GE, Chua M, Soiza RL. Prevalence of hyponatremia in elderly patients. J Am Geriatr Soc. 2006;54(9):1473; author reply-4. doi:10.1111/j.1532-5415.2006.00872.x.

3. Soiza RL, Cumming K, Clarke JM, Wood KM, Myint PK. Hyponatremia: special considerations in older patients. J Clin Med. 2014;3(3):944-58. doi:10.3390/jcm3030944.

4. Chua M, Hoyle GE, Soiza RL. Prognostic implications of hyponatremia in elderly hospitalized patients. Arch Gerontol Geriatr. 2007;45(3):253-8. doi:10.1016/j.archger.2006.11.002.

5. Wald R, Jaber BL, Price LL, Upadhyay A, Madias NE. Impact of hospital-associated hyponatremia on selected outcomes. Arch Intern Med. 2010;170(3):294-302. doi:10.1001/archinternmed. 2009.513

6. Waikar SS, Mount DB, Curhan GC. Mortality after hospitalization with mild, moderate, and severe hyponatremia. Am J Med. 2009;122(9):857-65. doi:10.1016/j.amjmed.2009.01.027. 
7. Liamis G, Milionis H, Elisaf M. A review of drug-induced hyponatremia. Am J Kidney Dis. 2008;52(1):144-53. doi:10. 1053/j.ajkd.2008.03.004.

8. Urso C, Brucculeri S, Caimi G. Acid-base and electrolyte abnormalities in heart failure: pathophysiology and implications. Heart Fail Rev. 2015;20(4):493-503. doi:10.1007/s10741-0159482-y.

9. de Groot V, Beckerman H, Lankhorst GJ, Bouter LM. How to measure comorbidity. a critical review of available methods. J Clin Epidemiol. 2003;56(3):221-9.

10. Gnjidic D, Cumming RG, Le Couteur DG, Handelsman DJ, Naganathan V, Abernethy DR, et al. Drug Burden Index and physical function in older Australian men. Br J Clin Pharmacol. 2009;68(1):97-105. doi:10.1111/j.1365-2125.2009.03411.x.

11. Kouladjian L, Gnjidic D, Chen TF, Mangoni AA, Hilmer SN. Drug Burden Index in older adults: theoretical and practical issues. Clin Interv Aging. 2014;9:1503-15. doi:10.2147/CIA. S66660.

12. Ramos-Levi AM, Duran Rodriguez-Hervada A, Mendez-Bailon M, Marco-Martinez J. Drug-induced hyponatremia: an updated review. Minerva Endocrinol. 2014;39(1):1-12.

13. Buon M, Gaillard C, Martin J, Fedrizzi S, Mosquet B, Coquerel A, et al. Risk of proton pump inhibitor-induced mild hyponatremia in older adults. J Am Geriatr Soc. 2013;61(11):2052-4. doi:10.1111/jgs.12534.

14. Hirokawa CA, Gray DR. Chlorpropamide-induced hyponatremia in the veteran population. Ann Pharmacother. 1992;26(10):1243-4.

15. Spital A. Diuretic-induced hyponatremia. Am J Nephrol. 1999;19(4):447-52.
16. Yamada H, Asano T, Aoki A, Ikoma A, Yoshida M, Kusaka I, et al. Combination therapy of angiotensin II receptor blocker and thiazide produces severe hyponatremia in elderly hypertensive subjects. Intern Med. 2014;53(7):749-52.

17. Izzedine H, Fardet L, Launay-Vacher V, Dorent R, Petitclerc T, Deray G. Angiotensin-converting enzyme inhibitor-induced syndrome of inappropriate secretion of antidiuretic hormone: case report and review of the literature. Clin Pharmacol Ther. 2002;71(6):503-7. doi:10.1067/mcp.2002.124520.

18. Sharma H, Pompei P. Antidepressant-induced hyponatraemia in the aged. Avoidance and management strategies. Drugs Aging. 1996;8(6):430-5.

19. Lindner G, Pfortmuller CA, Leichtle AB, Fiedler GM, Exadaktylos AK. Age-related variety in electrolyte levels and prevalence of dysnatremias and dyskalemias in patients presenting to the emergency department. Gerontology. 2014;60(5):420-3. doi:10. $1159 / 000360134$.

20. Tamma G, Goswami N, Reichmuth J, De Santo NG, Valenti G. Aquaporins, vasopressin, and aging: current perspectives. Endocrinology. 2015;156(3):777-88. doi:10.1210/en.2014-1812.

21. Kovesdy CP. Significance of hypo- and hypernatremia in chronic kidney disease. Nephrol Dial Transpl. 2012;27(3):891-8. doi:10. 1093/ndt/gfs038.

22. Kovesdy CP, Lott EH, Lu JL, Malakauskas SM, Ma JZ, Molnar $\mathrm{MZ}$, et al. Hyponatremia, hypernatremia, and mortality in patients with chronic kidney disease with and without congestive heart failure. Circulation. 2012;125(5):677-84. doi:10.1161/ circulationaha.111.065391. 